\title{
He-accreting WDs as SNe Ia Progenitors
}

\author{
Luciano Piersanti ${ }^{1}$, Amedeo Tornambé ${ }^{2}$, Lev Yungelson ${ }^{3}$, \\ and Oscar Straniero ${ }^{1}$ \\ ${ }^{1}$ INAF - Osservatorio Astronomico di Teramo, \\ via Mentore Maggini, snc, 64100, Teramo, ITALY \\ email: piersanti, straniero@oa-teramo.inaf.it \\ ${ }^{2}$ INAF - Osservatorio Astronomico di Roma, \\ via di Frascati, 33, 00040, Monteporzio Catone, ITALY \\ email: tornambe@oa-teramo.inaf.it \\ ${ }^{3}$ Institute of Astronomy, Moscow, RUSSIA \\ email: lev.yungelson@gmail.com
}

\begin{abstract}
We investigate the thermal response of CO WDs accreting He-rich matter directly from their companions in binary systems. Our results suggest that the He-channel cannot provide progenitors for the majority of "normal" SNe Ia.
\end{abstract}

Keywords. supernovae: general — white dwarfs — accretion

\section{Introduction}

Observational evidence clearly suggests that Type Ia supernovae (SNe Ia) are produced by the thermonuclear disruption of CO WDs accreting matter from their companions in binary systems. However, up to now no clear consensus exists concerning the nature of the donor. Currently the most favored scenarios involve either a normal star with a Hrich envelope [single degenerate scenario - Whelan \& Iben (1973)] or another CO WD [double degenerate scenario - Iben \& Tutukov (1984), Webbink (1984)]. An alternative evolutionary path is the so called He-donor channel; in this case He-rich matter is directly accreted from a He-star or from a He-WD. These objects are very interesting as, in principle, they could produce an explosion of SN Ia scale both via C-deflagration in a CO WD close to the Chandrasekhar limit and He-detonation in a sub-Chandrasekhar mass WD. Even if during the last decades He-detonation has not been considered promising as an SN Ia explosion mechanism [e.g. see Woosley \& Weaver (1994)], recently Sim et al. (2010) have demonstrated that, if the $\mathrm{CO}$ core is larger than $\sim 0.9 M_{\odot}$ and the He-buffer at the onset of the dynamical flash is small enough $\left(\leqslant 0.1 M_{\odot}\right)$, the resulting explosion could resemble all the observational properties of a "normal" SN Ia. Ruiter et al. (2011) have investigated by means of population synthesis technique the expected frequency of He-accreting WDs evolving into SN Ia-like events and they concluded that the subChandrasekhar "... model is the first model which demonstrates a sufficient number of SNe Ia events to account for all, or at least some substantial fraction of, SNe Ia ..., as well as two distinct formation channels with their own characteristic DTD."

Even if a large effort has been devoted in the past to investigating the thermal response of CO WDs directly accreting He-rich matter [a list of works dealing with this topic can be found in Shen \& Bilsten (2009)], a full evolutionary scenario is still missing. For this reason we computed a large set of fully evolutionary models of CO WDs in the mass range $0.6-1.1 M_{\odot}$ accreting He-rich matter with $\dot{M}$ in the range $10^{-9}-10^{-5} M_{\odot} \mathrm{yr}^{-1}$. The aim of our work is twofold: 1) the definition of the possible accretion regime, and 2) the final outcome of the accretion process. 




Figure 1. Possible accretion regimes in the $M_{W D}-\dot{M}$ plane.

\section{Results}

Our results are summarized in Figure 1 where we report as a function of the WD total mass and accretion rate the possible accretion regimes.

- RG Configuration: for very high value of the $\dot{M}$, but below the Eddington limit, the accretion rate is larger than the rate at which matter is converted into CO. Hence, a massive He-buffer forms and the WD expands to giant dimensions, overfilling its own Roche lobe. This implies that part of the transferred mass is ejected from the WD and it is lost from the binary system. The further evolution strongly depends on the amount of angular momentum carried away by the lost matter. If the ejected matter has the same specific angular momentum as the accretor, the mass transfer is dynamically stable but non conservative, while for larger values it becomes dynamically unstable and evolves in a common envelope. In the former case, part of the transferred mass is effectively accreted onto the WD, while in the latter the accumulation efficiency is practically zero $(\eta \simeq 0)$. This accretion regime occurs at the beginning of the mass transfer process when the donor is a He-WD or a massive He-star $\left(M_{H e-s t a r}>0.85 M_{\odot}\right)$.

- Steady Accretion: $\dot{M}$ is equal to the rate at which He is converted into CO. All the transferred mass is effectively accreted onto the WD which can grow in mass up to the Chandrasekhar limit.

- Mild Flashes: He-burning is almost switched off, a He-buffer is piled up and when it exceeds some critical value (depending both on $M_{C O}$ and $\dot{M}$ ) a He-flash is ignited. For $\dot{M}$ typical of this accretion regime the flash is mild and, hence, the expansion triggered by the sudden release of energy is small so that the WD remains well inside its own Roche lobe. As a consequence, the transferred matter is fully accumulated onto the WD.

- Strong Flashes: as in the previous accretion regime, the mass transfer proceeds through recurrent He-flashes, but in this case the energy delivered is so large that the accreting WD expands to giant dimension. As a consequence, a part of the previously accreted matter is ejected from the WD and lost by the system. The accumulation efficiency for this kind of systems is very low, but it could increase if the effects of strong radiation-driven wind are taken into account (Kato \& Hachisu 2004). For example, for a 




Figure 2. Mass extension of the He-buffer for a $0.8 M_{\odot} \mathrm{CO}$ WD accreting at various accretion rates. For more details see text.

model with $M_{\mathrm{WD}}=1.02 M_{\odot}$ and $\dot{M}=4 \times 10^{-7} M_{\odot} \mathrm{yr}^{-1}$ we obtain $\eta=0.11$ while Kato \& Hachisu (2004) provide $\eta=0.77$.

- He-Detonation: for $\dot{M} \leqslant 2 \times 10^{-8} M_{\odot} \mathrm{yr}^{-1}$, almost independent of the initial WD mass, the physical base of the He-rich zone is strongly degenerate and, when the accreted buffer exceeds a critical value, a dynamical He-flash is ignited. The resulting He-detonation triggers the explosion of the accreting WD. The mass extension of the Hebuffer is almost independent of the WD mass but it strongly depends on the accretion rate; lowering the accretion rate increases the mass extension of the He-buffer at the onset of the dynamical flash. This is shown in Figure 2 where we report for a $0.8 M_{\odot}$ CO WD the final mass at the explosion as a function of $\dot{M}$. Moreover, $\Delta M_{H e}$ strongly depends on the time evolution of $\dot{M}$, as shown in the same plot where we report the evolution of CO WD models accreting He-rich matter with a time-dependent law.

As we follow the long-term evolution of He-accreting WDs, we can also determine whether or not a given system will attain the Chandrasekhar limit, experiencing a Cdeflagration. In Figure 3 we report the profiles in the $\rho-T$ plane at the C-ignition for models accreting He-rich matter onto an initial $1.02 M_{\odot}$ CO WD at various rates, as labeled. Even if all the models have almost the same final mass $\left(M_{W D} \sim 1.36 M_{\odot}\right)$, for $\dot{M}=2 \times 10^{-6} M_{\odot} \mathrm{yr}^{-1}$ central C-ignition occurs, while for higher values C-burning is ignited off-center, very close to the stellar surface. In the former case a SN Ia event will result, while in the latter C-burning will propagate inward steadily, thus producing an ONeMg WD, which could eventually experience core collapse, thus forming a neutron star (Saio \& Nomoto 2004).

Our results suggest that the expected frequency of He-detonating systems with a large $\mathrm{CO}$ core and a small He-buffer is much lower than the one estimated by Ruiter and coworkers. In fact, the accumulation efficiency in the RG Configuration regime is smaller than 1, thus limiting the growth in mass of the CO core. Moreover, the mass extension of the He-buffer at the onset of the dynamical He-flash does depend on the accretion 


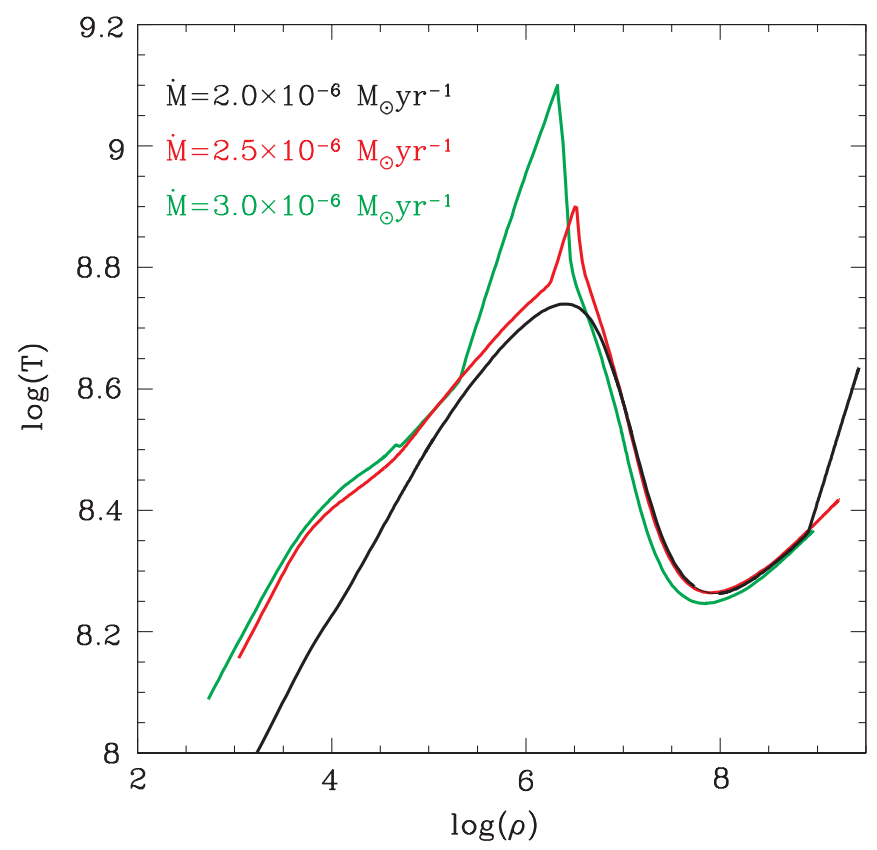

Figure 3. Profile in the $\rho-T$ plane at the C-ignition for models with different $\dot{M}$.

history. At the end, the area in the parameter space $M_{W D}-\dot{M}$ for which a CO WD could ignite C-burning at the center when it attains the Chandrasekhar limit is smaller than previously suggested (e.g. Nomoto 1982). In fact, for accretion rate larger than $2 \times 10^{-6} M_{\odot} \mathrm{yr}^{-1} \mathrm{C}$-burning is ignited off-center thus producing a collapsing $\mathrm{ONeMg}$ $\mathrm{WD}$, not an SN Ia event.

\section{References}

Iben, I. Jr \& Tutukov, A. V., 1984, ApJS, 54, 335

Kato, M. \& Hachisu, I., 2004, ApJ, 613, L129

Nomoto, K. I., 1982, ApJ, 253, 798

Ruiter, A. J., Belczynski, K., Sim, S.A., Hillebrandt, W., Fryer, C. L., Fink, M., \& Kromer, M., 2011 MNRAS, 417, 408

Saio, I. \& Nomoto, K. I., 2004, ApJ, 615,444

Shen, K. J. \& Bildsten, L., 2009, ApJ, 699, 1365

Sim, S. A., Röpke, F. K., Hillebrandt, W., Kromer, M., Pakmor, R., Fink, M., Ruiter, A. J., \& Seitenzahl, I. R., 2010, ApJ, 714, L52

Webbink, R. F., 1984, ApJ, 277, 355

Whelan, J. \& Iben, I. Jr, 1973, ApJ, 186, 1007

Woosley, S. E. \& Weaver, T. A., 1994, ApJ, 423, 371

\section{Discussion}

SIM: According to your results, what is the expected frequency of He-detonating WDs with a large $\mathrm{CO}$ core and small He-buffer?

PIERSANTi: I can not provide the exact value as we do not use our results as input for a population synthesis simulation, but I am quite confident that the frequency for "good" He-detonating WDs is at least a factor 50 (or more) smaller than the one estimated by Ruiter and coworkers. 